\title{
Erratum
}

\section{An upper bound on the energy of a gravitationally redshifted electron-positron annihilation line from the Crab pulsar}

\author{
P. S. Negi
}

Department of Physics, Kumaun University, Nainital 263 002, India

e-mail: negi@aries.ernet.in; psnegi_nainital@yahoo.com

A\&A 431, 673-677 (2005), DOI: 10.1051/0004-6361:20041790

Key words. gravitation - dence matter - equation of state - stars: neutron - errata, addenda

An error was found in the programming of the envelope model used in the paper. Some of the results obtained after correction differ significantly from those published in the paper, which should therefore be considered as erroneous throughout. The correct results will be published elsewhere. 